\title{
Miranda
}

Revue pluridisciplinaire du monde anglophone /

Multidisciplinary peer-reviewed journal on the English-

speaking world

$18 \mid 2019$

Guerre en poésie, poésie en guerre

\section{Exposition Vanité, identité, sexualité, Grayson Perry}

\section{Olivier Thircuir}

\section{QpenEdition}

\section{Journals}

Édition électronique

URL : http://journals.openedition.org/miranda/19570

DOI : $10.4000 /$ miranda. 19570

ISSN : 2108-6559

Éditeur

Université Toulouse - Jean Jaurès

Référence électronique

Olivier Thircuir, «Exposition Vanité, identité, sexualité, Grayson Perry », Miranda [En ligne], 18| 2019, mis en ligne le 17 avril 2019, consulté le 16 février 2021. URL : http://journals.openedition.org/miranda/ 19570 ; DOI : https://doi.org/10.4000/miranda.19570

Ce document a été généré automatiquement le 16 février 2021.

\section{$\Theta \Theta \Theta \Theta$}

Miranda is licensed under a Creative Commons Attribution-NonCommercial-NoDerivatives 4.0 International License. 


\title{
Exposition Vanité, identité, sexualité, Grayson Perry
}

\author{
Olivier Thircuir
}

213 avril - 2 septembre 2018

3 Paris, Musée de la Monnaie

419 octobre 2018 - 3 février 2019

5 Commissaire : Lucia Pesapane

\section{Craft of art}

6 L'exposition de Grayson Perry manifeste une réinvention de l'art décoratif pour servir une satire de la société britannique.

7 Patience et humilité sont siglées sur le réservoir rose bonbon du custom de Grayson Perry. Son deux-roues vrombit rive gauche de ses éclats acidulés. C'est une moto aux roues bleu électrique, percées de larges perforations, avec une généreuse selle de mobylette, frangée de lanières de cuir. Sur le porte-bagage, un reliquaire portatif pour accueillir Alan Measles, un doudou, fétiche, porteur depuis l'enfance des valeurs masculine de l'artiste qui se travestit pour dévoiler les ressorts d'une société en crise : vanité, masculinité et sexualité. C'est donc à moto que Grayson Perry braque, depuis le 10 octobre 2018, les valeurs de l'Hôtel de la Monnaie. Ce faisant, il propose des représentations désaxées de la souveraineté britannique, de ses majestés, de ses invisibilités, de ses non-dits, de ses fondements. On peut certes poser comme hypothèse que l'institution de la Monnaie frappe sur son avers les valeurs faciales d'un état, sur son revers le visage de son souverain. Ici le peuple, là, la reine. De son côté, Perry use de cet espace hautement symbolique qu'est la Monnaie pour proposer son droit de tirage (licence to kill contre un impérialisme guerrier), son droit de battre monnaie (feu sur l'ordre financier de la City), sa réflexion sur un code viril à dégonfler (vanité du mâle), sur une émission de valeurs (masculinité, sexualité) : Grayson Perry y oppose une 
histoire du Brexit, humble, touche à tout, un spectacle rebelle et pauvre, dans une série de contre-exemples qui glorifient une salutaire médiocrité, riche d'une culture aux hybridités plurielles, un éloge du common people.

\section{Fine arts rechapés}

8 Perry représente les paradoxes de la société britannique en utilisant des techniques modestes qui ont fait leurs preuves au Royaume-Uni depuis les inventions du mouvement art and craft. L'humilité et la patience s'exprime par la gravure, l'art de la tapisserie, ou l'art de la mode; mais la céramique aussi pour dégonfler nos petits volumes ou la sculpture en fonte, dont la rouille évoque une mémoire ouvrière, orpheline de ses modèles de vie et de ville.

9 La robe et le cape sont surpiquées de maints rapiéçages, boutonnées de symboles voyeurs, gonflées d'appendices mammaires bene pendentes : il s'agit de protéger l'exhibition de Claire, double travesti de Perry. Des robes de Claire qui travestissent l'artiste, déniaisent les pudeurs néoclassiques du l'amateur parisien autant que l'ego d'une capitale de la fashion. S'invente en ces bord de Seine, en face du Louvre, ain'it Mr. Ingres, une nouvelle odalisque de bande dessinée, gonades et pénis offerts à l'intaille xylographiée (Reclining Artist 1). Populaire, Perry préfère la robe de Françoise à la toilette de la duchesse de Guermantes. Le vêtement féminin hybride jouxte alors la nudité mâle pour proposer une masculinité féminisée et désarmée.

\section{Map of nowhere}

Sa tapisserie est une mappemonde de géographies autour desquelles pivotent les valeurs du millenium : elle récite un fourretout de slogans, un pot-pourri de références pour étudiant en cultural studies. Sur ce mille-fleurs de mots et de choses, l'aède craftsman tisse pour les lecteurs de Barthes une mythologie britannique: ses héros moyens ou bas, ses marques de bières, ses stadiums, ses chaînes de télévision, ses expressions creuses qui nouent la sociabilité d'un East-end façon nice cuppa tea. D'un autre côté, à l'opposé de l'exhibition du dérisoire, Perry file l'éloge de Julie Cope dans une hagiographie de la femme ordinaire, divorcée puis remariée, décédée trop jeune. Julie can cope, semble nous dire Perry, c'est-à-dire que seule, en tant que common woman, elle parvient à contenir les contradictions d'une société en forte mutation. À l'inverse, le tisserand de l'Essex démonte les algorithmes bancaires du yuppie blairien. Ainsi peut-on voir ce mythe dénigré de Tim Rockwell dans la grande série finale qui retrace l'ascension et la chute tragicomique d'un premier de cordée d'outre-Manche. Ce Tim Rockwell est un Richard Branson le petit de la computing technology: il incarne la laideur d'une classe arriviste, qui chasse à courre les places de l'aristocratie que l'on sait dégénérée. (The upper class at bay, 2012). Spoilons l'épilogue : tout ça finit mal, et pour cette bourgeoisie montante, et pour feue l'aristocratie dépouillée (\#Lamentation 1).

\section{Britain is best}

Enfin, l'art majeur de celui qui se définit comme un control freak: technique superbement maîtrisée qui a valu le Turner prize en 2003 à ce potier génial, le craftsman le plus doué de sa génération. Son art réinventé de la céramique souffle sur la vanité de 
l'esprit anglais, et pour recouvrir cette panse d'ale gonflée (Queen's Bitter), et pour moquer ce narcissisme jingoistic, par un jeu irisé de transparences délicates, à même de refléter les fantasmes d'une société toujours corsetée dans un syllabus érotique étriqué, comme au temps de Jane Austen. Car s'il s'inspire de l'art d'un Bernard Leach, il en renouvelle le classicisme formel par une abondance et un jeu de contraste où les incrustations polychromes exhibent les tensions sociales, anthropologiques et érotiques du royaume élizabéthain des années 2010. L'artiste s'y manifeste avec le plus de réussite : on y admire sa patience, son wit, un art de la couleur que le tissage généré par ordinateur ne peut égaler dans les tapisseries.

Pour circuler allègrement dans ce fatras de conflictualités, l'art de la customisation de la moto est donc le vecteur dégenré qui désigne un ennemi esthétique : l'académisme mâle et prétentieux des élites capitalistes dont les flux sont canalisés depuis la City. Cheers Mister Hirst! Avec cette exposition débordante, voici de la mécanique pour fluidifier la circulation des idées, de l'humour pour dérouter les humeurs, et de la semence faite à la main pour projeter, du royaume de Harvey au pays de Denis Papin, les liquides refroidis du fiasco néo-thatchérien. La cape et la chape dévoilent l'homo anglicus et ses sacrements blasphémés par un doudou divinisé. Sous les robes et les tapisseries de Perry se dévoile un artiste en Pénélope. C'est la kraft de Perry au sens d'une puissance, non pas souveraine, mais patiemment féminine et créatrice.

\section{INDEX}

Mots-clés : art, identité, nouvelle masculinité, hospitalité, sexualité, société, vanité, céramique, tapisserie

Keywords : art, identity, new masculinity, hospitality, sexuality, society, vanity, ceramic, tapestry

Thèmes : British art

\section{AUTEUR}

\section{OLIVIER THIRCUIR}

Agrégé externe de lettres modernes, collège G. Mélies, Paris.

Bachelor of Engineering, UCL ; professeur formateur, membre du GIPTIC de lettres.

olivier.thircuir@gmail.com 